\title{
Effects of Anti-TPO Antibodies on the Outcome of In Vitro Fertilization-Embryo Transfer Cycles
}

\author{
Bhagyashree M. Gadwal ${ }^{1}$, Deepika² ${ }^{2}$ Kamini Rao ${ }^{3}$ \\ ${ }^{1}$ Department of OBG (Reproductive Medicine), MRMC Kalaburagi. Karnataka, India. \\ 2, 3 Milann the Fertility Centre, Bengaluru, Karnataka, India
}

\section{ABSTRACT}

\section{BACKGROUND}

The objectives of the study were to investigate whether the incidence of antithyroid antibodies (ATAs) is related to an adverse outcome in the in-vitro fertilization and embryo transfer (IVF-ET) in terms of quality of oocytes retrieved, fertilisation, cleavage, embryo quality and implantation rate (primary outcome) as compared to patients without anti-thyroid antibodies and also evaluate the association of ATAs with abortion rate, biochemical pregnancy and clinical pregnancy (secondary outcome).

\section{METHODS}

A total of 52 women having anti-thyroid peroxidase antibody (anti- TPO Ab) level $\geq$ $35 \mathrm{IU} / \mathrm{mL}$ (Cases) and 21 women having anti-TPO Ab level < $35 \mathrm{IU} / \mathrm{mL}$ (Controls) undergoing IVF / ICSI from Jan 2014 to Dec 2014 at Milann - The Fertility Centre, Bangalore, were retrospectively analysed.

\section{RESULTS}

There was no difference among the cases and controls for the primary outcome, viz. maturation rate, fertilisation rate, cleavage rate, implantation rate, and embryo quality. However, the abortion rate and biochemical pregnancy rate were significantly higher in the cases than in controls $(\mathrm{P}=0.027$ for each parameter). On the other hand, the clinical pregnancy rate was significantly lower among the cases $(\mathrm{P}=0.045)$.

\section{CONCLUSIONS}

There was no statistically significant difference in the primary outcome among the two groups. The secondary outcome of abortion rate and biochemical pregnancy rate was significantly higher in the cases than controls. The clinical pregnancy rate was significantly lower among the cases as compared to controls.

Clinical Significance - Anti-TPO $\mathrm{Ab}$ acts as a prognostic factor and is disadvantageous to the pregnancy outcome following IVF-ET.

\section{KEY WORDS}

Abortion Rate, Clinical Pregnancy Rate, Fertilisation Rate, Implantation Rate, IVF/ICSI, Oocyte Maturation Rate
Corresponding Author: Dr. Deepika, C/o Dr Sharanprakash Patil, H.No 1-867/3/2, Behind Govt ITI College, Venkatesh Nagar, Kalaburagi - 585102, Karnataka, India.

E-mail:drdeepika@rediffmail.com

DOI: 10.14260/jemds/2021/706

How to Cite This Article:

Gadwal B, Deepika, Rao K. Effects of antiTPO antibodies on the outcome of in vitro fertilization-embryo transfer cycles. J Evolution Med Dent Sci 2021;10(39): 3487-3491, DOI:

10.14260/jemds/2021/706

Submission 22-06-2021, Peer Review 23-08-2021, Acceptance 30-08-2021, Published 27-09-2021.

Copyright (C) 2021 Bhagyashree M. Gadwal et al. This is an open access article distributed under Creative Commons Attribution License [Attribution 4.0 International (CC BY 4.0)] 


\section{BACKGROUND}

Anti-thyroid antibodies (ATAs) are autoantibodies directed in opposition to one or more components of the thyroid. Thyroid autoimmunity (TAI) has been associated with several untoward obstetric outcomes, such as preterm delivery, placental abruption, and low birth weight. Since the first report of the association of TAI with pregnancy loss in 1990, many studies have reported on the association; albeit with conflicting results. While several meta-analyses pointed towards the association of TAI with a higher threat of miscarriage in women who had conceived naturally, the relation of TAI with pregnancy loss in infertile women undergoing assisted reproductive technologies (ART) was unclear. $^{1}$ The causality and pathophysiology of this association, or the adequate course of treatment, have not been completely elucidated.

The most clinically pertinent anti-thyroid autoantibodies are anti-thyroid peroxidase antibodies (anti-TPO antibodies), thyrotropin receptor antibodies (TRAbs) and thyroglobulin antibodies. Of these, anti-TPO antibodies being the most common anti-thyroid autoantibody, are present in around 10 - $15 \%$ of sterile women. ${ }^{2}$

Anti-thyroid antibodies (ATAs) can interact with thyroid hormone receptors situated on the human oocyte and mar the chance of fertilization and subsequent embryo development. The presence of anti-thyroid antibodies in the endometrium has deleterious effects on embryo implantation and may lead to early pregnancy loss. ${ }^{3}$ Presence of ATAs in euthyroid women may be associated with fertility complications such as enhanced rate of abortion and occurrence of infertility. Women with TAI may undergo notable alterations in the levels of free thyroxine during ART and subsequent pregnancy, thereby hampering the normal genital tract functioning and foetal growth. ${ }^{2}$

In women undergoing assisted conception, ovarian stimulation adapted during IVF / ICSI has been shown to impair thyroid function, possibly exposing women with TAI to other risks. There is no consensus on the impact of ATA on the outcome of IVF-ET. Whether to initiate adjuvant therapy to control the thyroid auto-immunity before and during IVF is debatable. Our study intended to investigate whether the presence of anti-thyroid antibodies (ATAs) was related to an adverse outcome in IVF / ICSI.

\section{METHODS}

In this retrospective case-control study, patients receiving IVF / ICSI-ET in Milann-The Fertility Centre, Bengaluru, from Jan 2014 to Dec 2014 were included. The inclusion criteria were:

- Patients of known hypothyroidism.

- Young patients with poor ovarian reserve.

- Previous one or more IVF/ICSI-ET failures.

- Previous one or more abortions.

The exclusion criteria were - women with

- Other autoimmune diseases

- Anticardiolipin antibody

- Lupus anticoagulant

- Anti-nuclear antibody

\section{- Or rheumatoid factor}

The patients were divided into two groups:

1. Controls - Anti TPO Ab $<35 \mathrm{IU} / \mathrm{mL}$

2. Cases - Anti TPO $\mathrm{Ab} \geq 35 \mathrm{IU} / \mathrm{mL}$

\section{IVF-E T}

All the patients were administered a flexible antagonist protocol for stimulation.

\section{Collection of Clinical Information}

The clinical information, including age, body mass index (BMI), duration of infertility, basal serum levels of follicle stimulating hormone (FSH) and Luteinising Hormone (LH), was recorded. In addition, during the IVF treatment, data regarding some oocytes were retrieved, fertilisation rate, number of available embryos, number of embryos for transferring, gradation of embryos, pregnancy rate, implantation rate, clinical pregnancy rate and abortion rate were recorded and analysed.

Comparison of oocyte maturation rate, fertilization rate and implantation rate among cases and controls was the primary outcome.

Comparison of abortion rate, biochemical pregnancy rate and clinical pregnancy rate among cases and controls was the secondary outcome.

\section{Statistical Analysis}

Statistical analysis was done with SPSS version 13.0 statistics software package. Comparisons of quantitative data were performed with independent student's t-test between two groups, and those of qualitative data were carried out using a chi-square test. Alpha (significance level) was defined as 0.05 . A value of $P<0.05$ was regarded to be statistically significant.

\section{RESULTS}

\section{General Characteristics}

There were no significant differences in the age, duration of infertility, thyroid stimulating hormone (TSH), FSH and antimullerian hormone $(\mathrm{AMH})$ between the cases and controls. (Table 1)

\begin{tabular}{|ccccc|}
\hline Variables & Case & Control & t-Value & P-Value \\
Age & $32 \pm 3.6$ & $32.3 \pm 3.6$ & 0.416 & 0.678 \\
Duration of Infertility & $6.9 \pm 4.0$ & $7.2 \pm 4.2$ & 0.405 & 0.686 \\
TSH & $3.9 \pm 2.6$ & $3.1 \pm 1.6$ & 1.831 & 0.070 \\
FSH & $7.2 \pm 3.4$ & $6.7 \pm 2.9$ & 0.912 & 0.364 \\
AMH & $2.3 \pm 2.1$ & $2.8 \pm 3.1$ & 0.980 & 0.370 \\
\hline Table 1. General Characteristics and Hormonal Levels in Cases and \\
Controls \\
\hline
\end{tabular}

Since the cases and controls were not significantly different from each other with respect to the general characteristics, they were eligible to be compared for the primary and secondary outcomes. Based on statistical analysis, there were no significant differences in the maturation rate, fertilisation rate and cleavage rate among the cases and controls. (Table 2) 


\begin{tabular}{|cccc|}
\hline Variables & Case & Control & P-Value \\
Maturation rate & $74.1 \%(625 / 844)$ & $71.4 \%(297 / 401)$ & 0.750 \\
Fertilization rate & $75.0 \%(633 / 844)$ & $79.8 \%(322 / 416)$ & 0.622 \\
Cleavage rate & $92.4 \%(780 / 844)$ & $96.1 \%(400 / 416)$ & 0.440 \\
\hline Table 2. Controlled Ovarian Stimulation (COS) and IVF Outcome \\
\hline
\end{tabular}

The values of embryo grading were lower in controls than cases, though not significantly, as shown in Table 3.

\begin{tabular}{|cccc|}
\hline \multicolumn{4}{c|}{ Embryo Grading } \\
Embryo & Cases & Controls & P-Value \\
A & $53.0(67.1)$ & $28.0(66.7)$ & 0.912 \\
A+B & $5.0(6.3)$ & $4.0(9.5)$ & 0.479 \\
A+Blast & $8.0(10.1)$ & $1.0(2.4)$ & 0.106 \\
B & $8.0(10.1)$ & $4.0(9.5)$ & 0.930 \\
Blast & $5.0(6.3)$ & $5.0(11.9)$ & 0.027 \\
\hline Table 3. Frequency Distribution According to Embryo Grading \\
\hline
\end{tabular}

The implantation rate among cases and controls was not significantly different, as shown in Table 4.

\begin{tabular}{|lcccc|}
\hline \multicolumn{5}{|c|}{ Implantation Rate } \\
& Cases & Controls & Chi-Square & P-Value \\
\hline Yes & $29.0(36.7)$ & $18.0(42.9)$ & 0.436 & 0.77 \\
No & $50.0(63.3)$ & $24.0(57.1)$ & & \\
\hline \multicolumn{4}{|l}{ Table.4. Frequency } & Distribution According to Implantation Rate \\
\hline
\end{tabular}

The abortion rate and biochemical pregnancy rates were significantly higher $(\mathrm{P}=0.027)$ among cases than controls, as shown in Table 5. Conversely, the clinical pregnancy rate was significantly lower among the cases.

\begin{tabular}{|cccc|}
\hline Outcome & Cases & Controls & P-Value \\
Abortion rate & $7.0(24.1)$ & $1.0(5.6)$ & 0.027 \\
Biochemical pregnancy rate & $7.0(24.1)$ & $1.0(5.6)$ & 0.027 \\
Clinical pregnancy rate & $14.0(48.3)$ & $16.0(77.8)$ & 0.045 \\
\hline \multicolumn{2}{|c|}{ Table 5. Frequency Distribution According to Secondary Outcome } \\
\hline
\end{tabular}

\section{DISCUSSION}

It is well acknowledged in the published literature that thyroid malfunction could compromise fertility by numerous pathophysiological processes. Thyroid-related autoimmunity is a predominant cause of thyroid dysfunction. It has been shown that in women having TAI, the occurrence of infertility is very high. ${ }^{4}$ All the same; there is a lack of conclusive evidence for a causal relationship between TAI and infertility. Similarly, there is inadequate data on the direct effect of TAIs on IVF outcomes.

We compared the IVF outcome among cases (anti-TPO antibodies positive group) and controls (anti-TPO antibodies negative group) in the present study. Our results showed no difference among the cases and controls with respect to the primary outcome, viz. maturation rate, fertilisation rate, cleavage rate, implantation rate and quality of the embryo. However, the abortion rate and biochemical pregnancy rates were significantly higher in the cases than in the controls. In addition, the clinical pregnancy rate was significantly lower among the cases as compared to controls.

Kim et al. ${ }^{5}$ reported that the pregnancy rate was lower in anti-thyroid antibodies (ATA) positive infertile women as compared to infertile women who did not have ATAs $(26.3 \%$ vs. $39.3 \%$ ), which corroborated with our finding.

A study by Negro et al. ${ }^{6}$ concluded that in euthyroid women undergoing ART, the pregnancy and delivery rates were unaffected by the presence of TPO antibodies (TPOAb).
However, in TPOAb (+) group, high-normal TSH values were related to an enhanced threat of unsuccessful pregnancy or ensuing loss of pregnancy.

Medenica et al. ${ }^{7}$ probed the ART outcomes in 52 women. Of these, 26 women were TAI positive and 26 women were age and BMI-matched TAI negative controls. According to a personalised treatment schedule, two protocols were administered: either a long gonadotropin-releasing hormone agonist (GnRH-a) or a short GnRH-antagonist (GnRH-ant), along with urinary HMG and/or a recombinant FSH. 20/26 patients of the TAI positive group received treatment with levothyroxine for 19.5 months, with a mean daily dose of $67.49 \pm 29.40 \mathrm{mcg}$. The groups did not differ significantly with regard to the number of oocytes retrieved and fertilisation rates; an observation similar to ours. However, the rate of implantation was lesser in the TAI positive group than in controls $(\mathrm{P}=0.092)$ which is in contrast to our study. In addition, the rate of pregnancy for every initiated cycle was significantly different between the TAI positive and the TAI negative group (30.8 \% versus $61.5 \%$ ), $\mathrm{P}=0.026$. It was also significantly different between the TAI positive and the TAI negative group for embryo-transfer cycle and (34.8\% versus $66.7 \%$ ), $\mathrm{P}=0.029$. Based on multivariate analysis, the study concluded that women with TAI had a significantly lesser possibility to attain pregnancy (OR 0.036, $95 \% \mathrm{CI}$ [0.004-0.347], $\mathrm{P}=0.004$ ). This result is also consistent with our findings.

Zhong et al. ${ }^{3}$ retrospectively studied a total of 90 women with ATA and 676 infertile women without ATA, undergoing IVF / ICSI. The stimulation regimen of long GnRH-a was uniform in all patients. The total gonadotropin dose, days of ovarian stimulation, and the number of oocytes retrieved were not significantly different among the two groups. The fertilisation rate $(64.3 \%$ versus $74.6 \%, \mathrm{P}<.001)$ and implantation rate $(17.8 \%$ versus $27.1 \%, \mathrm{P}<.001)$ post-IVFET were considerably lower in the TAI positive group than in the TAI negative group as opposed to our study where we obtained similar fertilisation rates between the two groups. However, the lower pregnancy rate $(33.3 \%$ versus $46.7 \%$, P $=0.002$ ) and higher abortion rate reported in the ATA positive group ( $26.9 \%$ vs. $11.8 \%, \mathrm{P}=0.002)$ was in line with our finding of significantly lower CPR $(\mathrm{P}=0.045)$ and higher abortion rate in the cases $(\mathrm{P}=0.027)$. The authors did not mention the thyroid function in the study.

Similar to our study, Toulis et al. $(2010)^{8}$ reported that sub-fertile women with TAI undergoing IVF were at a significantly higher risk of pregnancy loss than women without TAI. However, the clinical pregnancy rates (CPR) and delivery rates were similar.

Thangaratinam et al. (2011) ${ }^{9}$ also found a significantly greater miscarriage rate amongst women with TAI, similar to our study. Though there was no reduction in CPR or implantation rates, women with TAI were found to have lower live birth rates.

In a meta-analysis of twelve studies between 1990 to 2015, Busnelli et al.10 tried to find the relation between thyroid auto immunity (TAI) and the IVF / ICSI cycle outcomes. Of the twelve studies, six were prospective while six were retrospective group studies. In comparison to TAI negative group women, TAI positive group women had a higher miscarriage rate (Odds Ratio 1.44; $95 \%$ Confidence Interval [1.06-1.95]; $\mathrm{P}=0.02 ; 12$ studies; 4876 women; $\mathrm{I}^{2}=$ 
$35 \%$ ), a lower live birth ratio (OR 0.73; $95 \%$ CI [0.54-0.99]; $\mathrm{P}=0.04 ; 9$ studies; 4396 women; $\mathrm{I}^{2}=41 \%$ ), a similar number of oocytes (standardized mean difference [SMD] 0.10; $95 \%$ CI [ -0.09 to 0.29 ]; $\mathrm{P}=0.28 ; 5$ studies; 1506 women; $\mathrm{I}^{2}=47$ $\%$ ), a similar clinical pregnancy rate (OR 0.90; $95 \%$ CI [0.771.06]; $\mathrm{P}=0.22 ; 12$ studies; 4876 women; $\mathrm{I}^{2}=7 \%$ ), a similar fertility rate (OR 1.11; $95 \%$ CI [0.97-1.27]; $\mathrm{P}=0.13 ; 3$ studies; 1082 women; $\mathrm{I}^{2}=0 \%$ ) and a similar implantation rate (OR 0.98; $95 \%$ CI [0.73-1.32]; $\mathrm{P}=0.91 ; 2$ studies; 918 women; $\left.\mathrm{I}^{2}=0 \%\right)$. The authors concluded that TAI does not affect IVF / ICSI outcome with regard to the number of oocytes retrieved and the chances of fertilisation, implantation and clinical pregnancy. They opined that the presence of thyroid autoantibodies may adversely affect the progression of a pregnancy, leading to an enhanced threat of miscarriage and a reduced chance of live birth. This is in line with our findings.

In contrast, Leiva et al.11 meta-analysis that included four papers between 1999 to 2017 failed to find an association between TAI and higher risk of reproductive loss, RR $=0.94$ $95 \%$ confidence interval: $0.71-1.24 ; \mathrm{P}=0.879$. Thus, the authors opined that the presence of anti-thyroid antibodies should be considered as a secondary biomarker of autoimmune disease rather than an actual cause of miscarriage in patients undergoing ART.

A meta-analysis by He et al. ${ }^{1}$ in 2016 included four definitive studies with data on 1855 infertile women, including 292 women with ATAs and 1563 women without ATAs. The study concluded that thyroid autoimmunity per se does not adversely affect ART outcomes in women without subclinical hypothyroidism. ${ }^{1}$

A meta-analysis by Poppe et al. ${ }^{12}$ retained four studies involving 1855 ICSI cycles. 290 women were TAI positive and 1565 women were TAI negative. In women with a clinical pregnancy, there was no difference in miscarriage among the 114 women with TAI and 651 women without TAI. Combined odds ratio $0.95,95 \% \mathrm{CI}$ [0.48 to 1.87]. Also there was no difference in the live birth rates among the two groups: OR $1.12,95 \% \mathrm{CI}$ [0.62 to 2.0]. The study concluded that TAI positive infertile women treated with ICSI were not at a greater risk of a first-trimester miscarriage in comparison to TAI negative women, which contrasts with our findings. The study authors hence concluded that the presence of TAI may become a new indication for ICSI, irrespective of the cause of infertility since it obviates the harmful impact of TAI on embryo quality.

In a systematic review of studies published between 2006 and 2018 by Simopoulou et al. ${ }^{5}$ fourteen studies reported clinical pregnancy rates. Eight of the studies were retrospective and the remaining five prospective. Only three studies observed a statistically significant lower clinical pregnancy rate in the thyroid auto antibodies positive group. These findings corroborate the results of three previous meta-analyses done in the field. ${ }^{10,1,8}$

A recent meta-analysis of 14 studies reported up to 2020 by Venables et al. ${ }^{13}$ suggested that TAI does not affect pregnancy outcomes in women undergoing IVF either overall or in euthyroid women.

The process of implantation is a critical step encompassing the interaction between the embryo and uterine epithelium. In the process, two immunologically and genetically different tissues have to communicate successfully. ${ }^{4}$ Considering that the clinical pregnancy rate (CPR) in our study was significantly lower in cases, it is likely that circulating anti-TPO antibodies act as a marker of a general immune imbalance affecting the maternal-foetal immunological relationship. Possibly, the thyroid antibodies have a detrimental effect on the trophoblastic cells, restraining their activity and hampering the natural growth of the foetus. Matalon et al. ${ }^{14}$ demonstrated proof of crossreactivity of thyroid autoantibodies and trophoblast antigens in the murine model, increasing foetal wastage with lower foetal and placental weights. A mice model study reported that anti-TPO-antibodies affected the development of the embryo post-implantation, resulting in foetal loss. ${ }^{15} \mathrm{We}$ conclude that anti-TPO antibodies act as a prognostic factor for ART outcomes. Therefore, their presence is damaging to the pregnancy outcome in the wake of IVF-ET. Further studies are required to ascertain the possible benefits of levothyroxine in such patients before IVF-ET.

\section{Clinical Significance}

Anti-TPO Ab acts as a prognostic factor and is detrimental to the pregnancy outcome following IVF-ET.

\section{CONCLUSIONS}

There was no statistically significant difference in the primary outcome among the two groups. The secondary outcome of abortion rate and biochemical pregnancy rates were significantly higher in the cases than controls. The clinical pregnancy rate was significantly lower among the cases as compared to controls.

Data sharing statement provided by the authors is available with the full text of this article at jemds.com.

Financial or other competing interests: None.

Disclosure forms provided by the authors are available with the full text of this article at jemds.com.

\section{REFERENCES}

[1] He H, Jing S, Gong F, et al. Effect of thyroid autoimmunity per se on assisted reproduction treatment outcomes: a meta-analysis. Taiwanese J Obs Gynaeol 2016;55:159e65.

[2] Poppe K, Velkeniers B. Thyroid and infertility. Verh K Acad Geneeskd Belg 2002;64(6):389-99.

[3] Zhong YYY, Wu H, Zhou C, et al. Relationship between antithyroid antibody and pregnancy outcome following in vitro fertilisation and embryo transfer. Int J Med Sci 2012;9(2):121-5.

[4] Simopoulou M, Sfakianoudis K, Maziotis E, et al. The impact of autoantibodies on ivf treatment and outcome: a systematic review. Int J Mol Sci 2019;20:892-900.

[5] Kim C, Chae H, Kang B, et al. Influence of antithyroid antibodies in euthyroid women on in vitro fertilizationembryo transfer outcome. Am J Reprod Immunol 1998;40:2-8.

[6] Negro R, Formoso G, Coppola L, et al. Euthyroid women with autoimmune disease undergoing assisted 
reproduction technologies: the role of autoimmunity and thyroid function. J Endocrinol Invest 2007;30(1):3-8.

[7] Medenica S, Garalejic E, Arsic B, et al. Follicular fluid thyroid autoantibodies, thyrotropin, free thyroxine levels and assisted reproductive technology outcome. PLoS One 2018;13(10): e0206652.

[8] Toulis KA, Goulis DG, Venetis CA, et al. Risk of spontaneous miscarriage in euthyroid women with thyroid autoimmunity undergoing IVF: a meta-analysis. Eur J Endocrinol 2010;162(4):643-52.

[9] Thangaratinam S, Tan A, Knox E, et al. Association between thyroid autoantibodies and miscarriage and preterm birth: meta-analysis of evidence. $\mathrm{Br}$ Med J 2011;342:d2616.

[10] Busnelli A, Paffoni A, Fedele L, et al. The impact of thyroid autoimmunity on IVF/ICSI outcome: a systematic review and meta-analysis. Human Reproduction Update 2016;22(6):793-4.

[11] Leiva P, Schwarze JE, Vasquez $P$, et al. There is no association between the presence of anti-thyroid antibodies and increased reproductive loss in pregnant women after ART: a systematic review and metaanalysis. JBRA Assisted Reproduction 2017;21(4):361-5.

[12] Poppe K, Autin C, Veltri F, et al. Thyroid autoimmunity and ICSI pregnancy outcomes. J Clin Endocrinol Metab 2018;103(5):1755-66.

[13] Venables A, Wong W, Way $M$, et al. Thyroid autoimmunity and IVF/ICSI outcomes in euthyroid women: a systematic review and meta-analysis. Reproductive Biol Endocrinol 2020;18:120.

[14] Matalon ST, Blank M, Levy Y. The pathogenic role of antithyroglobulin antibody on pregnancy: evidence from an active immunization model in mice. Hum Reprod 2003;18(5):1094-9.

[15] Lee YL, Ng HP, Lau KS, et al. Increased fetal abortion rate in autoimmune thyroid disease is related to circulating TPO autoantibodies in an autoimmune thyroiditis animal model. Fertil Steril 2009;91(Suppl 5):2104-9. 